International Journal of Pure and Applied Mathematics

Volume 97 No. $4 \quad 2014,431-445$

ISSN: 1311-8080 (printed version); ISSN: 1314-3395 (on-line version)

url: http://www.ijpam.eu

doi: http://dx.doi.org/10.12732/ijpam.v97i4.5

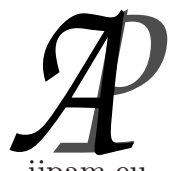

ijpam.eu

\title{
A NEW NUMERICAL INTEGRATOR FOR THE SOLUTION OF GENERAL SECOND ORDER ORDINARY DIFFERENTIAL EQUATIONS
}

\author{
A.O. Adesanya ${ }^{1 \S}$, J. Sunday ${ }^{2}$, A.A. Momoh ${ }^{1}$ \\ ${ }^{1}$ Department of Mathematics \\ Modibbo Adama University of Technology \\ Yola, Adamawa State, NIGERIA \\ ${ }^{2}$ Department of Mathematical Sciences \\ Adamawa State University \\ Mubi, Adamawa State, NIGERIA
}

\begin{abstract}
This paper considered the development of numerical integrator for the solution of second order initial value problems. The method was derived through the interpolation and collocation of the basis polynomial which is combination of power series and exponential function to derive a continuous linear multistep method. The method was implemented in block method which gave solution at a non overlapping interval. The method was found to be convergence and A stable. The efficiency of the method was tested on some numerical examples.
\end{abstract}

AMS Subject Classification: 65L05, 65L06, 65D30

Key Words: A-stable, interpolation, collocation, basis polynomial, block method, convergence, stability interval, non-overlapping

Received: January 29, 2014

(C) 2014 Academic Publications, Ltd. url: www.acadpubl.eu

${ }^{\S}$ Correspondence author 


\section{Introduction}

This paper considers the numerical solution to general second order initial value problem of the form

$$
y^{\prime \prime}=f\left(x, y, y^{\prime}\right), y\left(x_{n}\right)=y_{n}, \quad y^{\prime}\left(x_{n}\right)=y_{n}^{\prime}
$$

where $x_{n}$ is the initial point, $y\left(x_{n}\right)$ is the solution at the initial point, $f$ is continuous within the interval of integration and assumed to satisfied the existence and uniqueness theorem stated below

Theorem 1. (see [1]) Let

$$
u^{(n)}=f\left(x, u, u^{\prime}, \ldots, u^{(n-1)}\right), \quad u^{(k)}\left(x_{0}\right)=c_{k},
$$

$k=0,1, \ldots,(n-1)(u$ and $f$ are scalars $)$. Let $R$ be the region defined by the inequalities $x_{0} \leq x \leq x_{0}+a,\left|s_{j}-c_{j}\right| \leq b, j=0,1, \ldots,(n-1)(a>0 . b>0)$. Suppose the function $f\left(x, s_{0}, s_{1}, \ldots, s_{n-1}\right)$ is defined in $\Re$ and in addition:

(a) $f$ is non negative and non decreasing in each of $x, s_{0}, s_{1, \ldots}, s_{n-1}$ in $\Re$;

(b) $f\left(x, c_{0}, c_{1}, \ldots, c_{n-1}\right)>0$, for $x_{0} \leq x \leq x_{0}+a$, and

(c) $c_{k} \geq 0, k=0,1, \ldots, n-1$.

Then the initial value Problem 2 has a ubique solution in $\Re$.

See [1] for the proof.

Equation (1) are convectionally solved by reducing to systems of first order ordinary differential equation before appropriate method is applied. This method has been reported not to be efficient because it increases the dimension of the resulting systems of first order by the order of the differential equation; hence it wastes both the computer and human effort. Other setbacks of the method of reduction are discussed by $[2,3,4,5,6]$, consequently, direct method for the solution of (1) has arouse the interest of scholars.

Scholars have adopted method of interpolation and collocation of polynomial basis function to developed a continuous linear multistep method which can be implemented over an overlapping interval (predictor-corrector method) or non overlapping interval (block method). scholars reported that block method is better than the predictor corrector method because it is more efficient in terms of accuracy, cost of implementation, time effectiveness and the flexibility of the derived method; hence scholars has proposed different numerical scheme implemented in block method using different polynomial basis function ranging 
from power series, Langrange polynomial, Chebychev polynomial to mention but few $([7,8,9,10])$.

It was discovered that most of this method are not adequate for problems which are non linear, oscillatory and stiff problems due to poor stability properties exhibited by these methods.

Definition 2. (see [1]) A problem is said to be stiff if the following condition are fulfilled:

(a) No solution component is unstable or equivalently, no eigenvalue of the jacobian matrix has a real part which is at all large and positive and at least some component is very stable. That is atleast one eigenvalue has a negative part which is negative and large.

(b) The solution is slowly varying with respect to the negative real part of the eigenvalues.

Definition 3. (see [11]) The initial value Problem 1 is considered to be stiff oscillatory if the eigenvalues $\left\{\lambda_{j}=u_{j}+i v_{j}, j=1(1) m\right\}$ of the Jacobian $J=\frac{\partial f}{\partial y}$ possess the following properties $u_{j}<0, j=1(1) m, \operatorname{Max} 1 \leq j \leq m\left|u_{j}\right|>$ $\operatorname{Max} i \leq j \leq m\left|u_{j}\right|$ or if the stiffness ratio satisfies $S=\operatorname{Max} i, j\left|\frac{u_{i}}{u_{j}}\right|>1$ and $\left|u_{j}\right|<\left|u_{i}\right|$ for at least pair of $j$ in $1 \leq j \leq m$.

It should be recalled that stiff initial value problems was first encountered in the study of motion of spring of varying stiffness, since then, most problem have been discovered to be stiff especially in kinematics, chemical reactions, process control and electrical circuit theory, see [12]. Also discribed stiffness of ordinary differential equations in a manner that Problem 1 possess some stiffness if $R_{e}\left(\lambda_{i}\right)<0$, where $\lambda$ is the eigenvalue of the problem.

In order to improve on the stability of k-step method, scholars introduced hybrid points; hence hybrid linear multistep method was proposed, [3]. Reported that the introduction of hybrid points improved both the stability and accuracy of the method, $[3,7]$. Proposed one step method for the solution of higher order ordinary differential equation and both concluded that the method gave better stability properties and more accurate than the k-step method. This method was able to circumvent the Dahlquist stability barrier. It should be reminded that the implicit Eulers method was first proposed to solve stiff problems and was concluded that schemes which possess the properties of Astability are suitable for stiff problems.

Definition 4. (see [12]) A numerical method is said to be A-stable if the whole of the left-half plane $\{z: \operatorname{Re}(z) \leq 0\}$ is contained in the region $\{z:|\operatorname{Re}(z) \leq 1|\}$, where $R(z)$ is called the stability polynomial of the method. 
In the quest for the method that gives better stability properties, [13] proposed an approximate solution which is a combination of power series and exponential function. It was discovered that the method gives an A-stable method irrespective of how the points are selected. In this paper, we combined power series and exponential function as our basis function to develop our method.

\section{Methodology}

We consider a polynomial basis function in the form

$$
y(x)=\sum_{j=0}^{r+s-2} a_{j} x^{j}+a_{r+s-1} e^{\alpha x}
$$

where $r$ and $s$ are the number of interpolation and collocation points respectively. $x^{j}$ is the polynomial basis function. $a_{j}^{\prime} s \in \Re$ are constants to be determined on the partition $\Delta_{v}: a=x_{0}<x_{1}<\ldots<x_{N}=b$ of the interval of integration $[a, b]$, with constant step size $h$ given by $h=x_{n+1}-x_{n} ; n=0,1, . ., N-1$. Substituting the second derivatives of (3) into (1) gives

$$
f\left(x, y, y^{\prime}\right)=\sum_{j=2}^{r+s-2} j(j-1) a_{j} x^{j-2}+\alpha^{2} a_{r+s-1} e^{\alpha x}
$$

Interpolation (3) at $x_{n}$ and collocating (4) at $x_{n+s}, s=0\left(\frac{1}{5}\right) 1$ gives a system of non linear equation which can be written compactly in the form

$$
f_{n+s}=\sum_{j=2}^{r+s-2} j(j-1) a_{j} x^{j-2}+\alpha^{2} a_{r+s-1} e^{\alpha x}
$$

and

$$
y_{n+r}=\sum_{j=0}^{r+s-2} a_{j} x^{j}+a_{r+s-1} e^{\alpha x}, \quad r=0 .
$$

Solving for the unknown constants $a_{j}^{\prime} s$ in (5) and (6) using Guassian elimination method and substituting into (3) gives an hybrid continuous linear multistep method of the form

$$
y(t)=\alpha_{0} y_{n}+\alpha_{\frac{1}{5}} y_{n+\frac{1}{5}}+h^{2} \sum_{j=0}^{1} \beta_{n+j} f_{n+j}+\beta_{n+v} f_{n+v}, v=\frac{1}{5}\left(\frac{1}{5}\right) \frac{4}{5},
$$


where $t=\frac{x-x_{n}}{h}$,

$$
\begin{gathered}
\alpha_{0}=1-5 t \\
\alpha_{\frac{1}{5}}=5 t, \\
\beta_{0}=-\frac{1}{50400}\left(31250 t^{7}-131250 t^{6}+223125 t^{5}-196875 t^{4}+95900 t^{3}\right. \\
\left.-25200 t^{2}+2462 t\right), \\
\beta_{\frac{1}{5}}=\frac{1}{10080}\left(31250 t^{7}-122500 t^{6}+1863755 t^{5}-134750 t^{4}+42000 t^{3}-863 t\right), \\
\beta_{\frac{2}{5}}=-\frac{1}{25200}\left(156250 t^{7}-568750 t^{6}+774375 t^{5}-468125 t^{4}+105000 t^{3}-1522 t\right), \\
\beta_{\frac{3}{5}}=\frac{1}{25200}\left(156250 t^{7}-525000 t^{6}+643125 t^{5}-341250 t^{4}+70000 t^{3}-941 t\right), \\
\beta_{\frac{4}{5}}=-\frac{1}{50400}\left(156250 t^{7}-481250 t^{6}+538125 t^{5}-266875 t^{4}+52500 t^{3}-682 t\right), \\
\beta_{1}=\frac{1}{50400}\left(31250 t^{7}-87500 t^{6}+91875 t^{5}-43750 t^{4}+8400 t^{3}-107 t\right) .
\end{gathered}
$$

Evaluating (7) and it first derivative at $t=\frac{2}{5}\left(\frac{1}{5}\right) 1$, and $0\left(\frac{1}{5}\right) 1$ respectively, solving for the independent solution at the selected grid points gives a discrete block method in the form

$$
A^{(0)} Y_{m}^{(i)}=\sum_{i}^{1} h^{(i)} e_{i} y_{n}^{(i)}+h^{2}\left[d_{i} f\left(y_{n}\right)+b_{i} F\left(Y_{m}\right)\right], \quad i=0,1,
$$

where

$$
\begin{gathered}
Y_{m}=\left[\begin{array}{lllll}
y_{n+\frac{1}{5}} & y_{n+\frac{2}{5}} & y_{n+\frac{3}{5}} & y_{n+\frac{4}{5}} & y_{n+1}
\end{array}\right]^{T}, \\
F\left(Y_{m}\right)=\left[\begin{array}{lllll}
f_{n+\frac{1}{5}} & f_{n+\frac{2}{5}} & f_{n+\frac{3}{5}} & f_{n+\frac{4}{5}} & f_{n+1}
\end{array}\right]^{T}, \\
y_{n}^{(i)}=\left[\begin{array}{lllll}
y_{n-1}^{(i)} & y_{n-2}^{(i)} & y_{n-3}^{(i)} & y_{n-4}^{(i)} & y_{n}^{(i)}
\end{array}\right]^{T}, \\
F\left(y_{n}\right)=\left[\begin{array}{lllll}
f_{n-1} & f_{n-2} & f_{n-3} & f_{n-4} & f_{n}
\end{array}\right]^{T} .
\end{gathered}
$$

$A^{(0)}=5 \times 5$ identity matrix, when $i=0$,

$$
e_{0}=\left[\begin{array}{ccccc}
0 & 0 & 0 & 0 & 1 \\
0 & 0 & 0 & 0 & 1 \\
0 & 0 & 0 & 0 & 1 \\
0 & 0 & 0 & 0 & 1 \\
0 & 0 & 0 & 0 & 1
\end{array}\right], \quad e_{1}=\left[\begin{array}{ccccc}
0 & 0 & 0 & 0 & \frac{1}{5} \\
0 & 0 & 0 & 0 & \frac{2}{5} \\
0 & 0 & 0 & 0 & \frac{3}{5} \\
0 & 0 & 0 & 0 & \frac{4}{5} \\
0 & 0 & 0 & 0 & 1
\end{array}\right]
$$




$$
\begin{gathered}
d_{0}=\left[\begin{array}{ccccc}
0 & 0 & 0 & 0 & \frac{1231}{126000} \\
0 & 0 & 0 & 0 & \frac{71}{3150} \\
0 & 0 & 0 & 0 & \frac{123}{3500} \\
0 & 0 & 0 & 0 & \frac{376}{7875} \\
0 & 0 & 0 & 0 & \frac{61}{1008}
\end{array}\right], \\
b_{0}=\left[\begin{array}{ccccc}
\frac{863}{50400} & \frac{-761}{63000} & \frac{941}{126000} & \frac{-341}{126000} & \frac{107}{252000} \\
\frac{544}{7875} & \frac{-37}{1575} & \frac{136}{7875} & \frac{-101}{15750} & \frac{8}{7875} \\
\frac{3501}{28000} & \frac{-9}{3500} & \frac{87}{2800} & \frac{-9}{875} & \frac{9}{5600} \\
\frac{1424}{7875} & \frac{176}{785} & \frac{608}{7875} & \frac{-16}{1575} & \frac{16}{7875} \\
\frac{475}{2016} & \frac{25}{504} & \frac{125}{1008} & \frac{25}{1008} & \frac{11}{2016}
\end{array}\right],
\end{gathered}
$$

When $i=1$ :

$$
\begin{gathered}
e_{1}=\left[\begin{array}{lllll}
0 & 0 & 0 & 0 & 1 \\
0 & 0 & 0 & 0 & 1 \\
0 & 0 & 0 & 0 & 1 \\
0 & 0 & 0 & 0 & 1 \\
0 & 0 & 0 & 0 & 1
\end{array}\right], \quad d_{1}=\left[\begin{array}{ccccc}
0 & 0 & 0 & 0 & \frac{19}{288} \\
0 & 0 & 0 & 0 & \frac{14}{225} \\
0 & 0 & 0 & 0 & \frac{51}{850} \\
0 & 0 & 0 & 0 & \frac{14}{225} \\
0 & 0 & 0 & 0 & \frac{19}{280}
\end{array}\right], \\
b_{1}=\left[\begin{array}{cccccc}
\frac{1427}{7200} & \frac{-133}{1200} & \frac{241}{3600} & \frac{-173}{7200} & \frac{3}{800} \\
\frac{43}{150} & \frac{7}{225} & \frac{7}{225} & \frac{-1}{75} & \frac{1}{45} \\
\frac{219}{80} & \frac{57}{400} & \frac{57}{400} & \frac{-21}{800} & \frac{3}{800} \\
\frac{64}{225} & \frac{8}{75} & \frac{64}{225} & \frac{14}{225} & 0 \\
\frac{25}{96} & \frac{25}{144} & \frac{25}{144} & \frac{25}{96} & \frac{19}{288}
\end{array}\right]
\end{gathered}
$$

\subsection{Implementation of the Method}

In order to implement the method, we propose a prediction equation of the form

$$
Y_{m}^{(0)}=e y_{n}+h^{2} \sum_{\lambda=0}^{e} \frac{\partial^{\lambda}}{\partial x^{\lambda}} f(x, y)_{\left(x_{0}, y_{0}, y_{0}^{\prime}\right)} .
$$

Substituting (9) into (8) gives

$$
A^{(0)} Y_{m}^{(i)}=\sum_{i}^{1} h^{(i)} e_{i} y_{n}^{(i)}+h^{2}\left[d_{i} f\left(y_{n}\right)+b_{i} F\left(Y_{m}^{(0)}\right)\right] .
$$

Hence, (10) represents our new method. 


\section{Analysis of Basic Properties of the Method}

\subsection{Order of the Block}

Let the linear operator $\ell\{y(x): h\}$ associated with the discrete block method (8) be defined as

$$
\ell\{y(x): h\}=A^{(0)} Y_{m}^{(i)}-\sum_{i}^{1} h^{(i)} e_{i} y_{n}^{(i)}-h^{2}\left[d_{i} f\left(y_{n}\right)+b_{i} F\left(Y_{m}\right)\right]
$$

Expanding (8) in Taylor series and comparing the coefficient of $h$ gives

$$
\ell\{y(x): h\}=C_{0} y(x)+C_{1} h y^{1}(x)+\ldots+C_{p} h^{p} y^{p}(x)+C_{p+1} h^{p+1} y^{p+1}(x)+\ldots .
$$

Definition 5. The linear operator $\ell$ and associated block formular are said to be of order $p$ if $C_{0}=C_{1}=\ldots C_{p}=C_{p+1}=0, C_{p+2} \neq 0 . C_{p+2}$ is called the error constant and implies that the truncation error is given by $t_{n+k}=$ $C_{p+2} h^{p+2} y^{p+2}(x)+0\left(h^{p+3}\right)$.

For our method, expanding (8) in Taylor series and comparing the coefficient of $h$ gives the error constant as

$$
\begin{aligned}
& C_{8}= \\
& {\left[\begin{array}{lllll}
-2.1058(-8) & -5.1471(-8) & -8.0571(-8) & -1.0836(-7) & -1.455(-7)
\end{array}\right]^{T} .}
\end{aligned}
$$

\subsection{Zero Stabilty of the Method}

Definition 6. A block method is said to be zero stable if as $h \rightarrow 0$, the roots, $r_{j}=1(1) k$ of the first characteristics polynomials $p(x)=0$ that is $p(r)=\operatorname{det}\left[\sum A^{(0)} R^{k-1}\right]=0$ satisfying $|R| \leq 1$ must have multiplicity equal to unity.

For our method:

$$
p(R)=\left[R\left[\begin{array}{lllll}
1 & 0 & 0 & 0 & 0 \\
0 & 1 & 0 & 0 & 0 \\
0 & 0 & 1 & 0 & 0 \\
0 & 0 & 0 & 1 & 0 \\
0 & 0 & 0 & 0 & 1
\end{array}\right]-\left[\begin{array}{lllll}
0 & 0 & 0 & 0 & 1 \\
0 & 0 & 0 & 0 & 1 \\
0 & 0 & 0 & 0 & 1 \\
0 & 0 & 0 & 0 & 1 \\
0 & 0 & 0 & 0 & 1
\end{array}\right]\right]=0
$$

$p(R)=R^{4}(R-1)=0 \Rightarrow R=0,0,0,0,1$.

Hence, the new block integration is zero-stable. 


\subsection{Consistency}

The block integrator (8) is consistent since it has order $p=6$.

\subsection{Convergence}

Definition 7. (see [3]) A method is said to be convergence if $\lim _{h \longrightarrow 0} y\left(x_{n}\right)=$ $y_{n}$ where $y\left(x_{n}\right)$ is the approximate solution, $y_{n}$ is the analytical solution.

Theorem 8. The necessary and sufficient conditions that a continuous linear multistep method be convergentare that it must be consistent and zerostable.

\subsection{Region of Absolute Stability}

Definition 9. Region of absolute stability is a region in the complex $Z$-plane, where $\tau=\lambda h . \tau$ is defined as those values of $Z$ such that the numerical solution of $y^{\prime \prime}=-\lambda^{2} y$ and $y^{\prime}=-\lambda y$ satisfies $y_{j} \rightarrow 0$ as $j \rightarrow \infty$ for any initial value condition.

We adopted the boundary locus method to determine the stability of our method. Substituting $y^{\prime \prime}=-\lambda^{2} y, y^{\prime}=-\lambda y$ into (7) gives the stability region as shown in Figure 1.

\section{Numerical Examples}

Problem 10. We consider the mildly stiff problem given by

$$
y^{\prime \prime}=-1001 y^{\prime}-1000 y, y(0)=1, \quad y^{\prime}(0)=-1
$$

Exact solution $y(x)=\exp (-x)$.

This problem was solved by [14]. The result is shown graphically and in Figure 2 and tablulated in Table 1 . It should be noted that the maximum error was taken at $y(10.002)=4.5309220642(-5)$

Problem 11. We consider the non linear second order Van der pol equation

$$
y^{\prime \prime}+\mu\left(y^{2}-1\right) y^{\prime}+y=0, \quad y(0)=2, \quad y^{\prime}(0)=0 .
$$

This problem is known not to have analytical solution and is highly stiff if $\mu=1000$. We state the condition for finding the solution to this problem. 


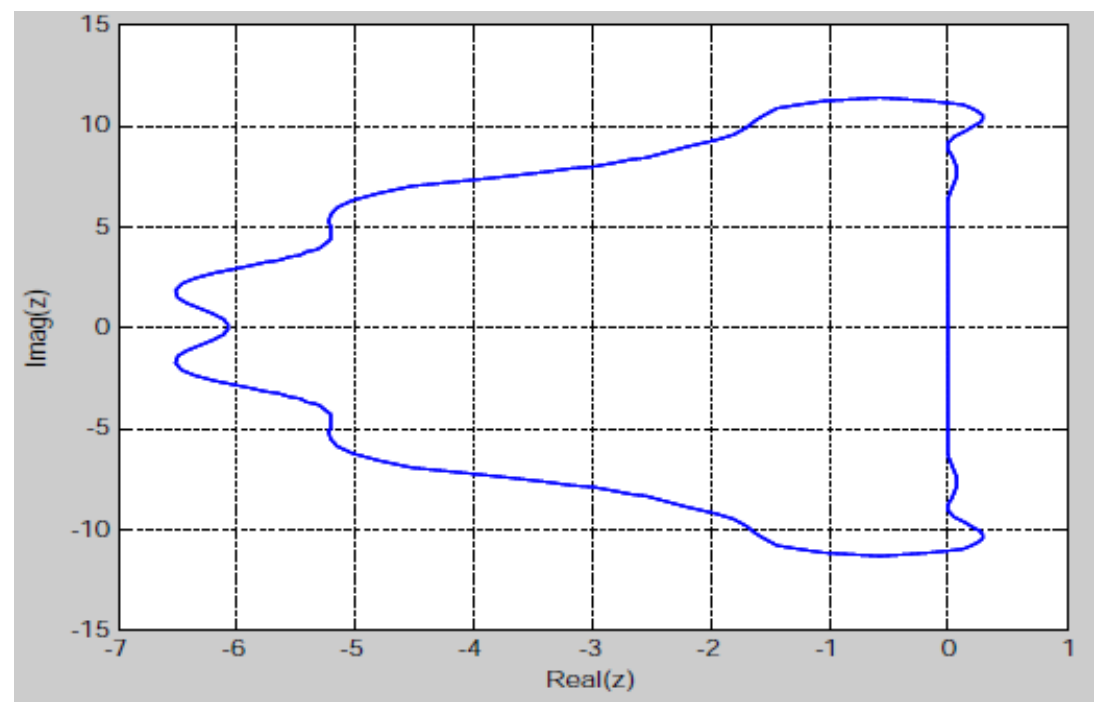

Figure 1: Stability region of the method

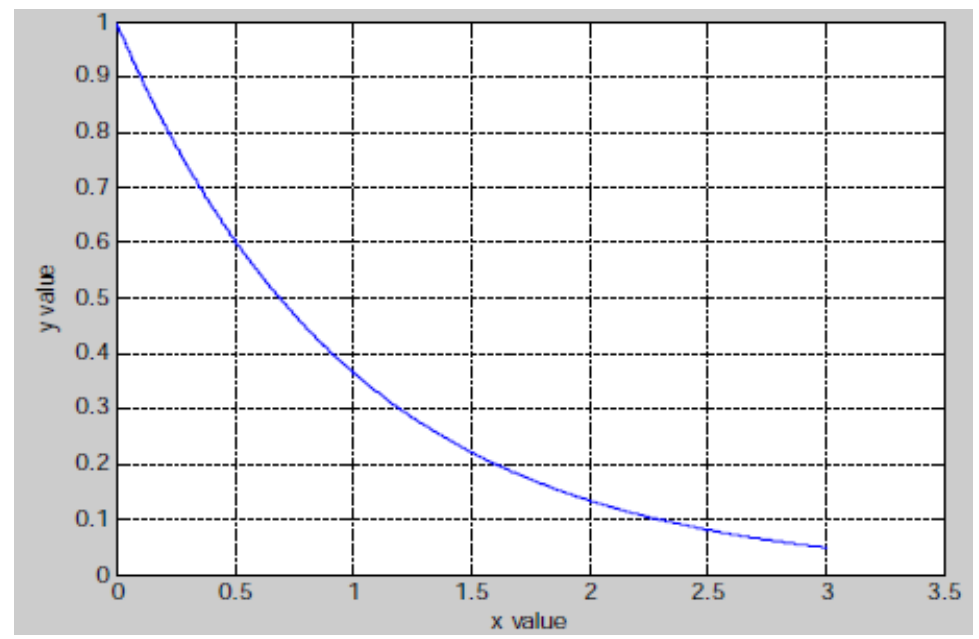

Figure 2: Result of Problem 1

Condition 12. Let $y\left(x_{n}\right)$ be the exact solution to the differential equation (1). Let $\underset{h \longrightarrow h_{i}}{\operatorname{Lim}} \Delta_{i} \longrightarrow y\left(x_{n}\right)$ and $\underset{h \longrightarrow h_{i}}{\operatorname{Lim}} \Delta_{i+1} \longrightarrow y\left(x_{n}\right)$ such that $\left|\Delta_{i+1}-\Delta_{i}\right| \longrightarrow$ 0 , then $\Delta_{i+1}$ is the numerical solution of (1) where $h_{i}>h_{i+1} \longrightarrow 0, i$ denote the stepsize and $n$ is the evaluation point ([5]) 


\begin{tabular}{ccc}
\hline$h$ & Computed result & Error \\
\hline 0.0032 & $4.5309220642(-5)$ & $2.61496(-17)$ \\
0.0030 & $4.5309220642(-5)$ & $1.19194(-17)$ \\
0.0025 & $4.5309220642(-5)$ & $6.99310(-18)$ \\
0.0020 & $4.5309220642(-5)$ & $3.79487(-19)$ \\
\hline
\end{tabular}

Table 1: Results of Problem 1

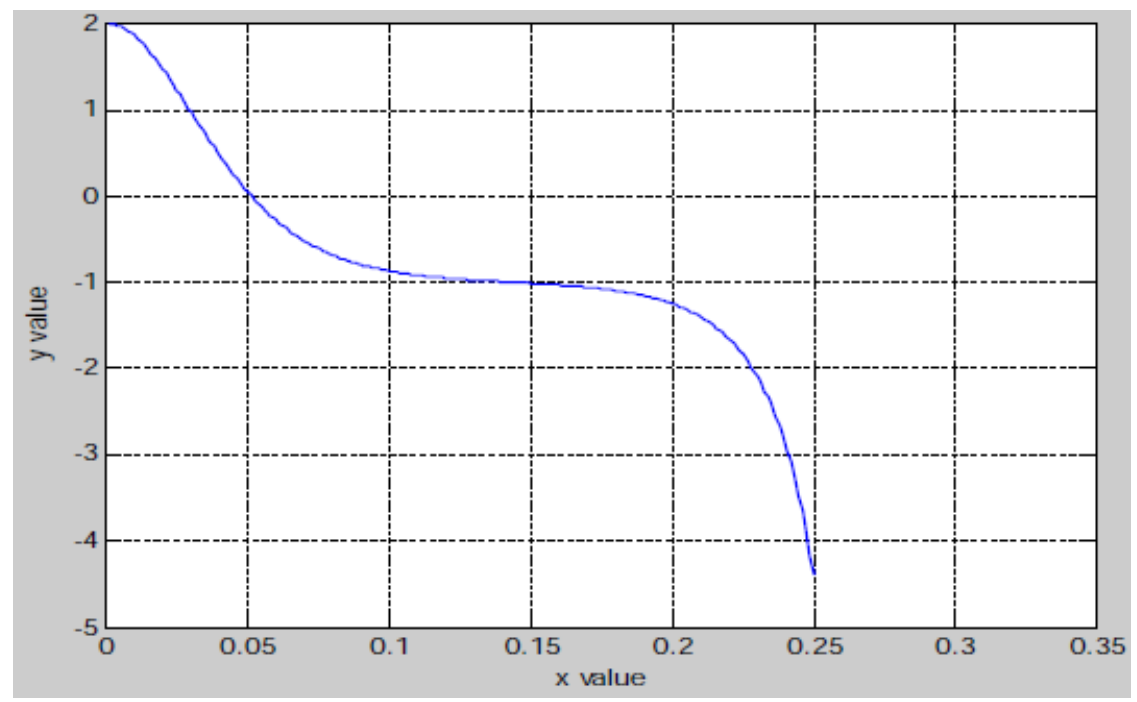

Figure 3: Result of Problem 2

\begin{tabular}{ccccc}
\hline $\mathrm{X}$ & \multicolumn{1}{c}{$\Delta_{1}$} & \multicolumn{1}{c}{$\Delta_{2}$} & \multicolumn{1}{c}{$\Delta_{3}$} & \multicolumn{1}{c}{$\Delta_{5}$} \\
\hline 0.02 & 1.471447914478928 & 1.471455980944556 & 1.471455980953264 & 1.471455980953267 \\
0.04 & 0.471522126413996 & 0.471537829080601 & 0.471537829087997 & 0.471537829087996 \\
0.06 & -0.282882467975155 & -0.282868614357267 & -0.282868614357701 & -0.282868614357707 \\
0.08 & -0.684779639126195 & -0.684765280195834 & -0.684765280205178 & -0.684765280205192 \\
0.10 & -0.869688408607038 & -0.869665997993542 & -0.869665998017794 & -0.869665998017818 \\
0.12 & -0.952128695721998 & -0.952081802206767 & -0.952081802264043 & -0.952081802264095 \\
0.14 & -0.994591259407622 & -0.994481675062548 & -0.994481675199508 & -0.994481675199639 \\
0.16 & -1.032114062340613 & -1.031848233831873 & -1.031848234165432 & -1.031848234165753 \\
0.18 & -1.097302863373722 & -1.096643772174615 & -1.096643773002270 & -1.096643773003067 \\
0.20 & -1.250533770686602 & -1.248846635886537 & -1.248846638005774 & -1.248846638007801 \\
\hline
\end{tabular}

Table 2: Results of Problem 2

The solution to Problem 2 is shown in Figure 3 and tabulated in Tables 2 and 3 . 


\begin{tabular}{cccc}
\hline$x$ & $\Delta_{2}-\Delta_{1}$ & $\Delta_{3}-\Delta_{2}$ & $\Delta_{4}-\Delta_{3}$ \\
\hline 0.02 & $8.0665(-06)$ & $8.7080(-12)$ & $3.0000(-15)$ \\
0.04 & $1.5703(-05)$ & $7.3960(-12)$ & $1.0000(-15)$ \\
0.06 & $1.4324(-05)$ & $4.3400(-13)$ & $6.0000(-15)$ \\
0.08 & $1.4359(-05)$ & $9.3440(-12)$ & $1.4000(-14)$ \\
0.10 & $2.2411(-05)$ & $2.4252(-11)$ & $2.4000(-14)$ \\
0.12 & $4.6894(-05)$ & $5.7276(-11)$ & $5.2000(-14)$ \\
0.14 & $1.0958(-04)$ & $1.3696(-10)$ & $1.3100(-13)$ \\
0.16 & $2.6583(-04)$ & $3.3356(-10)$ & $3.2100(-13)$ \\
0.18 & $6.59092(-4)$ & $8.2766(-10)$ & $7.9700(-13)$ \\
0.20 & $1.6871(-03)$ & $2.1192(-09)$ & $2.0270(-12)$ \\
\hline
\end{tabular}

Table 3: Results of Problem 3

\begin{tabular}{ccccc}
\hline $\mathrm{x}$ & Exact result & New result & Error & Error in[15] \\
\hline 1.1036 & 0.797152508880881 & 0.797152507062671 & $1.81821(-09)$ & $2.8047(-10)$ \\
1.2036 & 0.871118127112423 & 0.871118125001794 & $2.11062(-09)$ & $2.7950(-10)$ \\
1.3036 & 0.930288436749650 & 0.930288434445024 & $2.30194(-09)$ & $2.1490(-10)$ \\
1.4036 & 0.972304504262088 & 0.972304501871309 & $2.37077(-09)$ & $5.4975(-11)$ \\
1.5036 & 0.995491281635333 & 0.995491279245362 & $2.38997(-09)$ & $1.1545(-10)$ \\
1.6036 & 0.998924385222935 & 0.998924382899993 & $2.32294(-09)$ & $4.4825(-10)$ \\
1.7036 & 0.982466948018641 & 0.982466945799413 & $2.21922(-09)$ & $7.7969(-10)$ \\
1.8036 & 0.946775076109341 & 0.946775073999843 & $2.10949(-09)$ & $1.1840(-09)$ \\
1.9036 & 0.893271691795843 & 0.893271689775324 & $2.02051(-09)$ & $1.6318(-09)$ \\
2.0036 & 0.824089806171033 & 0.824089804200570 & $1.97046(-09)$ & $2.0567(-09)$ \\
\hline
\end{tabular}

Table 4: Results of Problem 3

Note that $\Delta_{1} \longrightarrow h=0.01, \Delta_{2} \longrightarrow h=0.001, \Delta_{3} \longrightarrow h=0.0001, \Delta_{4} \longrightarrow$ $h=0.00001$

Problem 13. We consider the non linear initial value problem

$$
y^{\prime \prime}=\frac{\left(y^{\prime}\right)^{2}}{2 y}-2 y, \quad y\left(\frac{\pi}{6}\right)=\frac{1}{4}, \quad y^{\prime}\left(\frac{\pi}{6}\right)=\frac{\sqrt{3}}{2}, \quad h=0.05 .
$$

Exact solution $y(x)=(\sin x)^{2}$.

This problem was solved by [5] where a five step method implemented in block method was proposed. The result is shown in Figure 4 and Table 4 . 


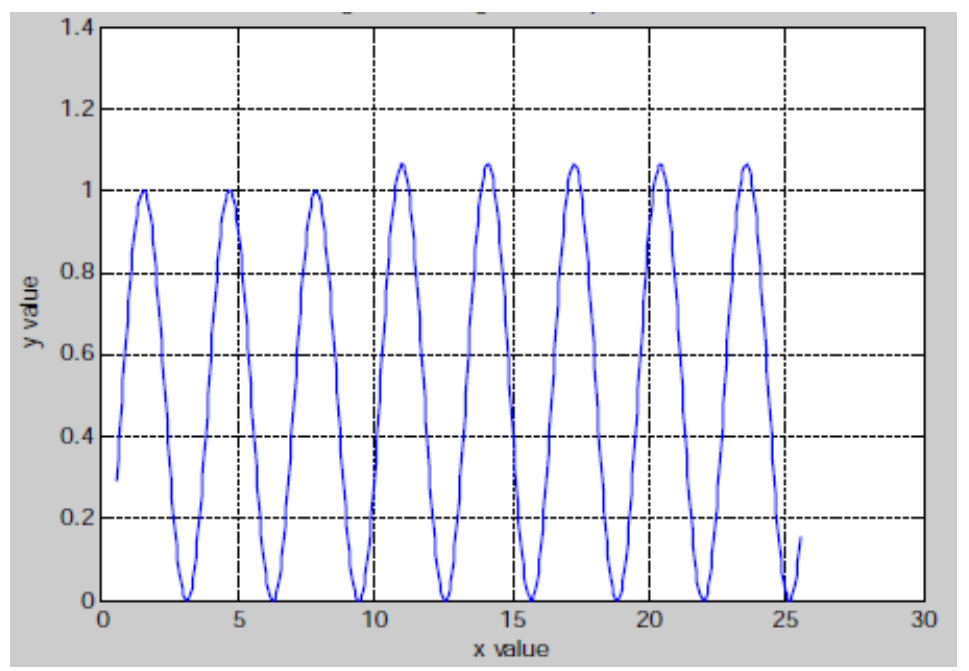

Figure 4: Result of Problem 3

\begin{tabular}{lccl}
\hline \multicolumn{1}{c}{$h$} & computed result & Error & EABPBCM \\
\hline 0.1 & 0.493150568758218 & $2.152032(-08)$ & - \\
0.05 & 0.493150589933258 & $4.452806(-10)$ & - \\
0.01 & 0.493150590278517 & $2.103873(-14)$ & $3.2211(-10)$ \\
\hline
\end{tabular}

Table 5: Results of Problem 4

Problem 14. We consider a highly oscillatory test problem

$$
y^{\prime \prime}+\lambda^{2} y=0, \quad y(0)=1, \quad y^{\prime}(0)=2, \quad \lambda=2 .
$$

Exact solution $y(x)=\cos 2 x+\sin 2 x$.

This problem was solved by [16] where a method fo order five implemented in block predictor-block corrector was proposed. The solution is shoem in Figure 5 and Table 4 . It should be noted that the result is taken at $x=1.0$, $y(1)=0.493150590278540$.

\subsection{Discussion of Result}

We have considered four numerical examples in this paper. The result as shown graphically in order to confirm if they conform with the exact solution. It should be noted that the method give better results if the value of $h \leq 0.0032$ for Problem 1. and Table 1 re-affirm the convergence of the method. $\Delta_{4}$ is 


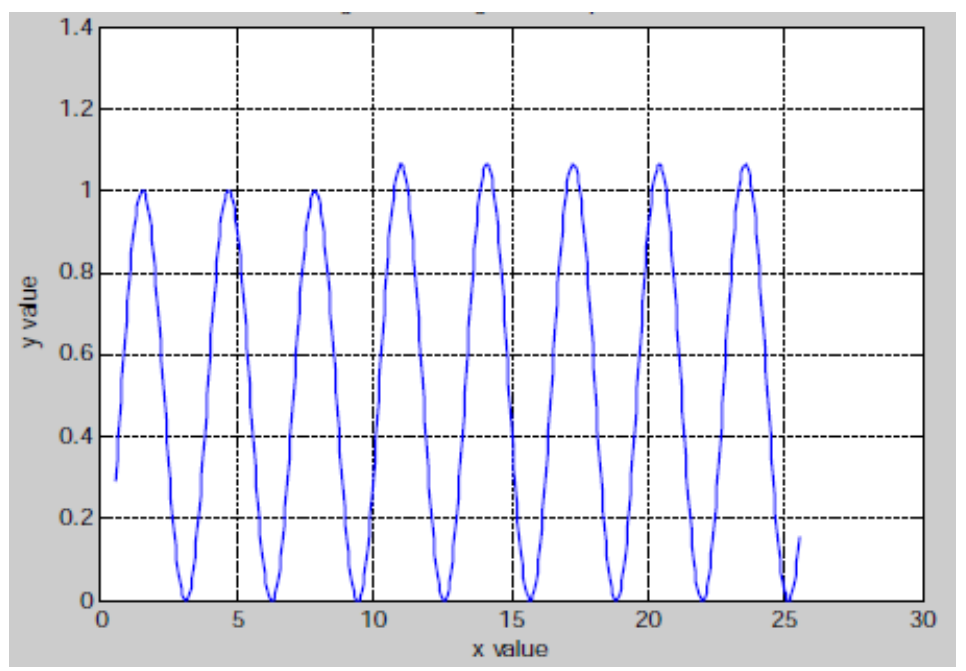

Figure 5: Result of Problem 4

the best approximation to Problem 2 as shown in the condition I and Table 3 also affirm the convergence of the method. Table 4 compared our results with the existing method and shown that our method has better stability and gives better approximation as is increasing. Table 5 shown that our method is better than the existing method we compared with at equally affirm the stability by varrying the value of $h$.

\section{Conclusion}

We have proposed a numerical method in this paper. The approximate solution is combination of power series and exponential function. The developed method has a better stability properties compared with the existing method as shown in the numerical example and compete favourably. It must be noted that the method proposed in this paper is not a self starting.

\section{References}

[1] V. V. Wend, Uniqueness of solution of ordinary differential equations, The American Monthly, 74(8), 1967, 27-33.

[2] A. A. James, A. O. Adesanya, J. Sunday, Countinous block method for the solution of second order initial value problem of ordinary differential 
equations. Intern. J. of Pure and Applied Mathematics, 88(3), 2013, 405416,doi:http://dx.doi.org/10.12732/ijpam.v8313.3

[3] T. A. Anake, D. O. Awoyemi, A. O. Adesanya, One step implicit hybrid block method for the direct solution of general second order ordinary differential equations. IAENG, Intern. J. of Applied mathematics, 42(4), 2012, IJAM. 42-4-04.

[4] J. Vigo-Aguilar, H. Ramos. Variable step size implementation of multistep method for $y^{\prime \prime}=f\left(x, y, y^{\prime}\right)$.J. of Comput. and Appl. Math. 192, 2006, 114-131.

[5] S. N. Jator, A six order linear multistep method for direct solution of $y^{\prime \prime}=f\left(x, y, y^{\prime}\right)$.Intern. J. of Pure and Applied Mathematics, 4(1), 2007, 457-472.

[6] D. O. Awoyemi, E. A. Adebile, A. O. Adesanya, T. A. Anake, Modified block method for the direct solution of second order ordinary differential equations, Intern. J. of Applied Mathematics and Computations, 3(3), 2011, 181-188.

[7] A. O. Adesanya, M. R. Odekunle, M. K. Fasasi, Continous hybrid block method for the solution of general third ordinary differential equations. Intern. J. of Computational and Applied Mathematics, 8(3) 2013, 225-236.

[8] B. I. Zarina, S. Mohammed, I. Kharol, M. Zanariah, Block method for generalized multistep Adams Method and backward differentiation formula in solving third order ODEs, Mathematika, 2005, 25-33.

[9] S. J. Kayode, A. A. Adeyeye, 3-step hybrid method for direct solution of second order initial value problems. Aust. J. of Basic and Applied Sciences, $5(2), 2011,2121-2126$.

[10] Z. A. Majid, P. S. Phang, M. Suleiman, Direct two point block method, one step method for solving general second order ordinary differential equations, Mathematical Problem in Engineering 12, article ID 184253, 2012, doi: 10.1155/2012/18425 (2011).

[11] P. W. Gaffery, A performance evaluation of some FORTRAIN subroutine for the solution of stiff oscillatory ODEs, ACM Transaction of Mathematical Software, 10, 1984, 58-72. 
[12] J. D. Lambert, Computational method in ordinary differential equation, John Wiley, New York.

[13] J. Sunday, M. R. Odekunle, A. O. Adesanya, Order six block integrator for the solution of first-order differential equations, Intern. J. of Mathematics and Soft Computing, 3(1), 2012, 87-96.

[14] S. N. Jator, On a class of hybrid method for $y^{\prime \prime}=\left(x, y, y^{\prime}\right)$. Intern. J. of Pure and Applied Mathematics, 59(4), 2010, 381-395.

[15] A. O. Adesanya, D. O. Udoh, A. M. Ajileye, A new hybrid block method for the solution of general third order initial value problem of ordinary differential equations, Intern. J. of Pure and Applied Mathematics, 86(2), 2013, 365-375. doi:http://dx.doi.org.10.2732/ijpam.v86i2.11

[16] A. O. Adesanya, M. R. Odekunle, M. A. Alkali, Three steps block predictor-corrector method for the solution of general second order ordinary differential equations. Intern. J. of Eng. Research and Application, 2(1), 2012, 2297-2301. 
